It should be borne in mind in relation to these figures that the age given is the age of death and not the age of the incidence of the ulcer, which must be considered as occurring earlier.

In regard to size, Dr. Osler mentions an ulcer $19 \mathrm{~cm}$. by $10 \mathrm{~cm}$., reported by Peabody, as the largest one he knows of. The largest one in my series (No. 28) was $9 \mathrm{~cm}$. to $10 \mathrm{~cm}$. by $8 \mathrm{~cm}$. to $10 \mathrm{~cm}$. The same authority refers to a case reported by Berthold in which there were 34 small ulcers. Similar cases of multiple ulcers are seen in my cases Nos. 10 and 32 above.

It should be remembered in basing conclusions on the above figures that the lame, the halt, and the blind of the city's streets sooner or later find their way to the Philadelphia Hospital and Almshouse, and that it is pre-eminently the house of chronic invalidism and disease.

Dr. Joseph Walsh, late pathologist to the Henry Phipps Institute, tells me that during his term of service (1903) he made autopsies on 52 cases dying of tuberculosis of the lungs, and that in one case there were numerous small superficial ulcers in the stomach. Dr. Rosenberger, who followed him, says that during the year 1904 there were 55 autopsies, and no instance of gastric or duodenal ulceration.

To briefly recapitulate, the total number of medical admissions to the Philadelphia Hospital for ten years from January 1, 1893, to December 31, 1902, inclusive, was 39,542. The total number of autopsies was 2830 . The total number of ulcers was 42 , of which 2 were purely duodenal.

So that the summary, including the figures given by Dr. Howard for the Pennsylvania and University Hospitals, is as follows:

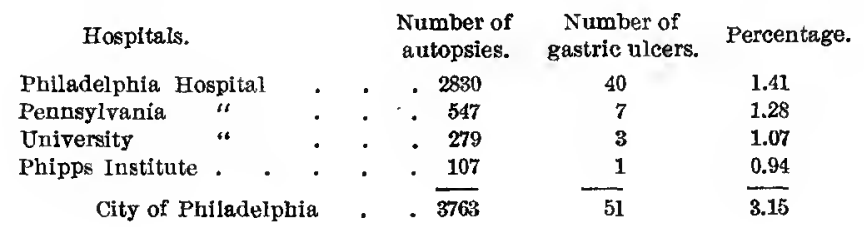

\title{
REPORT OF FIFTY CASES OF APPENDICITIS AND FIFTY CASES OF HERNIA WITH REFERENCE TO ALBUMINURIA.
}

\author{
By F. E. Bunts, M.D., \\ OF CLEVELAND, OHIO.
}

IN a paper read by Dr. John C. Munro before the American Surgical Association, in 1893, he calls attention to the significance of albumin and casts in surgical patients. It is particularly of interest 
to note that he states that approximately 35 per cent. of all patients treated in the surgical ward showed signs of renal trouble. In concluding his paper he says: "We should expect evidence of renal irritation in over a third of the surgical cases found in a municipal hospital. The mere presence of a trace of albumin, with or without hyaline and granular casts, unattended by other evidence of renal damage, should not influence the prognosis in surgical disease or operation; and furthermore albumin and casts alone are apparently no contraindication to the administration of ether."

For some time my attention has been particularly drawn to the common occurrence of albumin and casts in the urine of appendicitis cases, so common, indeed, was this that I had been forced to feel that there was a definite relation between appendicitis and albuminuria and had in preparation a paper upon this subject. The statistics collected by Dr. Munro, however, made it appear that it was so common in all surgical cases that I concluded to take two classes of operations in which the peritoneum was opened, in one of which inflammatory processes were or had been present, and the other in which such was not the case. As a type of the first class I have taken 50 consecutive cases of appendicitis, and for the second class have selected 50 consecutive cases of hernia, none of which were the seat of inflammation.

These 100 cases may be summarized in the following manner: Appendicitis, 50 cases; of these, 43 were operated upon during the attack, and 7 between attacks, with 2 deaths. Examination of the urine showed albumin present in 18 cases previous to operation, disappearing after operation in 5 cases. Twenty patients who had previously been free from albuminuria developed it subsequent to the operation, making a total of 33 , in whom it was present subsequent to operation.

Hernia (non-inflammatory), 50 cases; no deaths; of these, 11 showed albumin in the urine before operation, disappearing after operation in but 2 cases. Eleven new cases of albuminuria developed subsequent to operation, making a total of 20 in whom it was then present.

The number is obviously too small to have more than passing significance, and yet several interesting facts present themselves, and, should the collation of more numerous cases of a similar nature show similar results, might be of some value.

It may be said that these cases, with two or three exceptions, occurred in my service at Charity Hospital, and the examinations of the urine were made by one of the resident staff, assigned to that duty for four months at a time, and all doubtful examinations were subject to the approval or disapproval of the resident pathologist.

Referring to the tables, we find that of the inflammatory cases, 18 , or 36 per cent., had albumin before operation, while of the non- 
inflammatory cases, but 22 per cent. had albumin; 13 , or 26 per cent., of the former had it both before and after, and 18 of the latter were similarly affected. Of the former, the albumin disappeared in 28 per cent. after operation, while in the latter it disappeared in but 18 per cent.

These observations, if they show anything, would tend toward the belief that intraperitoneal inflammation increases the frequency of the appearance of albumin in the urine in about 18 per cent. of cases; that operations in this class of cases cause the development of albuminuria in 40 per cent. of new cases, and an improvement or disappearance of albuminuria in 10 per cent.

In the non-inflammatory class, operations are followed by the development of new cases of albuminuria in but 18 per cent., and of disappearance of albumin in but 4 per cent. The smaller percentage cured by operation in the latter group suggests that the presence of albumin in non-inflammatory cases is more liable to be a permanent condition than in inflammatory cases.

It is a somewhat startling fact that 66 per cent. of all inflammatory cases operated upon had albuminuria subsequently, and 60 per cent. of these did not have albumin previous to operation. There is also a very decided difference between the percentage of albuminuria cases in inflammatory and non-inflammatory cases, both bcfore and after operation. The statistics are very meagre, but they suffice to emphasize the observations in the paper already alluded to, that albumin is found in a very large percentage of surgical cases; that it cannot be considered on any ground yet established that it is of itself a contraindication to operation, for in the 100 cases here reported there were only 2 deaths, both acute suppurative cases, and among the cases of recovery from operation was a woman seven months pregnant, with albumin and hyaline casts, who was subsequently delivered of a full-term healthy child.

Unfortunately, there has been no systematic record kept of the condition of the urine at the time patients have left the hospital, so that no data can be offered at present as to the disappearance or continuance of the albumin and casts, but from records kept in a few scattered cases, I am of the opinion that a large number of them ultimately show no trace of kidney disturbance where such disturbance could originally reasonably be ascribed to the changes brought about by the surgical disease for which they were operated. While most of these cases have been operated under ether anæsthesia, it is a well-known fact that chloroform anæsthesia is followed not infrequently by the appearance of albumin in the urine. 